\title{
Depression and Anxiety
}

SHORT COMMENTARY

\section{Pseudodepression as an Anticipatory Symptom of Frontal Lobe Brain Tumors \\ Domenico Chirchiglia*}

Department of Neurosurgery, University of Catanzaro, Italy

*Corresponding author: Domenico Chirchiglia, MD, Department of Neurosurgery, University of Catanzaro, Campus Germaneto, VLE Europa, 88100, Catanzaro, Italy, Tel: +3909613647410

\begin{abstract}
Frontal brain tumors often cause psychiatric disorders related to the functions of the prefrontal cortex. The prefrontal cortex expresses cognitive functions, which are superior functions concerning personality, conception, planning, ability to manage social relations. The dorsolateral part of the prefrontal cortex, if damaged, causes a depressive symptomatology, consisting of apathy, mood reduction and other symptoms. Kleist defined depression secondary to brain tumors, pseudodepression syndrome. It is a rare condition associated to brain frontal tumors, the depression that occurs first and not vice versa. The reasons are not clear. However, it is important to control depressed patients over the years, they must perform neuroimaging tests, avoiding the late discovery of a brain frontal tumor.
\end{abstract}

\section{Keywords}

Pseudodepression, Depression, Frontal lobe, Frontal tumors

It is known that depression is one of the symptoms of a frontal brain tumor. This is because the frontal cortex, or rather prefrontal, represents cognitive functions, such as behavior, mood, personality. Depression may be one of the symptoms of a frontal brain tumor. Depression secondary to organic diseases is called pseudodepression, a term coined by Karl Kleist in 1934. The dorsolateral region of the prefrontal cortex is responsible for the pseudodepression syndrome which is characterized by "negative symptoms" such as apathy, lack of concentration, tendency to isolate. When the prefrontal dorsolateral part is affected by tumor, surgical excision, if possible, also eliminates depressive signs [1]. The literature is rich in this association and many cases are described, while rare cases have been found in literature where depression anticipates the onset of frontal brain tumors. In these cases depression can be the only symptom. What is the explanation? A response may be the consolidation of a depression diagnosis, so the affected subject does not carry out neuroimaging examinations for years, especially if it does not report other symptoms. If a symptom such as headache appears, the depressed person is subjected to brain CT or $\mathrm{MRI}$, and discovers that he has a brain tumor. In the literature, the case of a depressed patient, affected by thyroid carcinoma, is reported, who, at the onset of headache, performed neuroimaging tests that showed the presence of a cerebral metastasis [2].

What consideration should be made? Time is an important variable, especially in medicine, because it allows us to anticipate the discovery of a disease, by operating the diagnostic means. Often this does not happen or happens with delay, as in the case of depressed subjects who concentrate on the disease and do not perform for long periods of time, diagnostic tests, and when they perform, they risk finding a brain tumor. Therefore, to be suffering from depression, does not mean that, for years controls should not be carried out, because a brain CT or MRI are the only tool able to unravel if over the years a tumour mass has formed inside the brain. Other studies argue that the anguish of the depressive syndrome may be a risk factor for the development of brain tumors, especially if there is familiarity. The role of the doctor: the doctor must supervise carefully the patient depressed, must monitor it, advise the execution of neuroimaging tests periodically. This is important, because there are slow-growing brain 
tumors such as meningiomas, which are often asymptomatic, so the subject tends not to carry out control tests. Then, knowing already to suffer from tumor, has the advantage of intervening first and get better results. In short, depression, not as a symptom of frontal brain tumor, but as a anticipatory sign. It is important to know this eventuality, because it induces to carry out checks in the depressed patient, with the purpose of knowing that he is suffering only from depression and not discover anything else. Pseudodepression syndrome is typical of prefrontal tumours and is often the only symptom. Medical or surgical therapy is used to control the disease or, by removing the tumor, eliminate it $[3,4]$. At the same time, we need to know that depression, as a disease, can anticipate the presence of a frontal tumor, without which we do not know. What are the reasons, we must be prepared to monitor the depressed patient, through the execution of neuroimaging examinations. This is the right way to anticipate the discovery of a brain tumor, and that it is not the depression that delays the discovery. An important aspect concerns the appearance of neurological signs in the depressed patient. The appearance of a simple headache that becomes persistent, must alarm the patient, because the headache is one of the signs, although nonspecific, of the presence of a brain tumor. Therefore, if the depression, which can in turn cause headache, headache is added, you have to proceed with the investigation. Pseudode- pression syndrome can be the only symptom and early sign of a frontal tumor [5-7]. Current knowledge is a valuable help in implementing diagnostic schemes to preserve the lives of these subjects.

\section{References}

1. Ozdilek B, Midi L (2011) Brain tumor presenting with psychiatric symptoms. J Neuropsychiatry Clin Neurosci 23: E43-E44.

2. Oreskovic NM, Strother CG, Zibners LM (2007) An unusual case of a central nervous system tumor presenting as a chief complaint of depression. Pediatr Emerg Care 23: 486-488.

3. Moise D, Madhusoodanan S (2006) Psychiatric symptoms associated with brain tumors: A clinical enigma. CNS Spectr 11: 28-31.

4. Subramoniam Madhusoodanan, Mark Bryan Ting, Tara Farah, Umran Ugur (2015) Psychiatric aspects of brain tumors: A review. World J Psychiatry 5: 273-285.

5. Huang J, Zeng C, Xiao J, Zhao D, Tang H, et al. (2017) Association between depression and brain tumor: A systematic review and meta-analysis. Oncotarget 8: 94932-94943.

6. Zimmaro LA, Sephton SE, Siwik CJ, Phillips KM, Rebholz WN, et al. (2018) Depressive symptoms predict head and neck cancer survival: Examining plausible behavioral and biological pathways. Cancer 124: 1053-1060.

7. Goebel S, Stark AM, Kaup L, von Harscher M, Mehdorn HM (2011) Distress in patients with newly diagnosed brain tumours. Psychooncology 20: 623-630. 